Research Article

\title{
Periodic Property and Asymptotic Behavior for a Discrete Ratio-Dependent Food-Chain System with Delays
}

\author{
Changjin Xu $\mathbb{D}^{1},{ }^{1}$ Peiluan Li $\mathbb{D}^{2},{ }^{2}$ and Maoxin Liao ${ }^{3}$ \\ ${ }^{1}$ Guizhou Key Laboratory of Economics System Simulation, Guizhou University of Finance and Economics, Guiyang 550004, \\ China \\ ${ }^{2}$ School of Mathematics and Statistics, Henan University of Science and Technology, Luoyang 471023, China \\ ${ }^{3}$ School of Mathematics and Physics, University of South China, Hengyang 421001, China \\ Correspondence should be addressed to Changjin Xu; xcj403@126.com
}

Received 19 February 2020; Revised 23 June 2020; Accepted 1 July 2020; Published 23 July 2020

Academic Editor: J. R. Torregrosa

Copyright (c) 2020 Changjin Xu et al. This is an open access article distributed under the Creative Commons Attribution License, which permits unrestricted use, distribution, and reproduction in any medium, provided the original work is properly cited.

In this paper, a discrete ratio-dependent food-chain system with delay is investigated. By using Gaines and Mawhin's continuation theorem of coincidence degree theory and the method of Lyapunov function, a set of sufficient conditions for the existence of positive periodic solutions and global asymptotic stability of the model are established.

\section{Introduction}

The past decades have witnessed a great deal of interest in the periodic phenomena of predator-prey systems. For example, Zhang and Tian [1] investigated the multiple periodic solutions of a generalized predator-prey system with exploited terms. Zhang and Wang [2] analyzed the existence and global attractivity of a positive periodic solution for a generalized delayed prey-predator system. Li et al. [3] studied multiple positive periodic solutions of $n$ species delay competition systems with harvesting terms. Ding et al. [4] made a detailed discussion on the periodic solution of a Gause-type predator-prey systems with impulse. Shen and Li [5] obtained a set of sufficient conditions for the existence of at least one strictly positive periodic solution and the uniqueness and global attractivity of positive periodic solution for an impulsive predator-prey model with dispersion and time delays. For more knowledge about the periodic solutions of predator-prey models, one can see [6-12]. For the papers mentioned above, it shall be pointed out that many investigations have been performed to analyze the dynamical behavior on biological species by using continuous or impulsive mathematical models [6-9]. It has been widely argued and accepted that difference equations often occur in numerous setting and forms, both in mathematics itself and in its applications to statistics, computing, electrical circuit analysis, dynamics, economics, biology, and other fields [13]. In recent years, $\mathrm{Xu}$ et al. [14] have studied the persistence and stability of the following ratio-dependent food-chain system with delay:

$$
\left\{\begin{array}{l}
\dot{x}_{1}(t)=x_{1}(t)\left[a_{1}-a_{11} x_{1}\left(t-\tau_{1}\right)-\frac{a_{12} x_{2}(t)}{m_{12} x_{2}(t)+x_{1}(t)}\right] \\
\dot{x}_{2}(t)=x_{2}(t)\left[-a_{2}+\frac{a_{21} x_{1}\left(t-\tau_{2}\right)}{m_{12} x_{2}\left(t-\tau_{2}\right)+x_{1}\left(t-\tau_{2}\right)}\right. \\
\left.-\frac{a_{23} x_{3}(t)}{m_{23} x_{3}(t)+x_{2}(t)}\right], \\
\dot{x}_{3}(t)=x_{3}(t)\left[-a_{3}+\frac{a_{32} x_{2}\left(t-\tau_{3}\right)}{m_{23} x_{3}\left(t-\tau_{3}\right)+x_{2}\left(t-\tau_{3}\right)}\right]
\end{array}\right.
$$


where $x_{1}(t), x_{2}(t)$, and $x_{3}(t)$ denote densities of the prey, predator, and the top predator populations at time $t$, respectively, $\tau_{1} \geq 0$ is constant time delay due to negative feedback of the prey, and $\tau_{2} \geq 0$ and $\tau_{3} \geq 0$ are constant time delays due to gestation. $a_{i}(i=1,2,3), a_{11}, a_{12}, a_{21}, a_{23}, a_{32}, m_{12}$, and $m_{23}$ are all positive constants. In detail, one can see [14].

In real life, many biological and environmental parameters do vary in time(for example, naturally subject to seasonal fluctuations). However, $\mathrm{Xu}$ et al. [14] did not involve the varying parameters of the food-chain model. To describe the object relationship between predator population and prey population, we modify system (1) as the following nonautonomous ratio-dependent food-chain system with varying delay:

$$
\left\{\begin{array}{l}
\dot{x}_{1}(t)=x_{1}(t)\left[a_{1}(t)-a_{11}(t) x_{1}\left(t-\tau_{1}(t)\right)-\frac{a_{12}(t) x_{2}(t)}{m_{12}(t) x_{2}(t)+x_{1}(t)}\right] \\
\dot{x}_{2}(t)=x_{2}(t)\left[-a_{2}(t)+\frac{a_{21}(t) x_{1}\left(t-\tau_{2}(t)\right)}{m_{12}(t) x_{2}\left(t-\tau_{2}(t)\right)+x_{1}\left(t-\tau_{2}(t)\right)}-\frac{a_{23}(t) x_{3}(t)}{m_{23}(t) x_{3}(t)+x_{2}(t)}\right] \\
\dot{x}_{3}(t)=x_{3}(t)\left[-a_{3}(t)+\frac{a_{32}(t) x_{2}\left(t-\tau_{3}(t)\right)}{m_{23}(t) x_{3}\left(t-\tau_{3}(t)\right)+x_{2}\left(t-\tau_{3}(t)\right)}\right]
\end{array}\right.
$$

Many authors [15-18] argue that discrete time models governed by difference equations are more appropriate to describe the dynamics relationship among populations than continuous ones when the populations have nonoverlapping generations. Moreover, discrete time models can also provide efficient models of continuous ones for numerical simulation.
In order to reveal the dynamic relationship of predator and prey and explain the stability law of both species by applying the computer simulations, we think that it is reasonable to study time ratio-dependent food-chain systems governed by difference equations. Following the lines of Wiener [19] and Fan and Wang [20], we obtain the discrete time analogue of system (2):

$$
\left\{\begin{array}{l}
x_{1}(k+1)=x_{1}(k) \exp \left\{a_{1}(k)-a_{11}(k) x_{1}\left(k-\tau_{1}(k)\right)-\frac{a_{12}(k) x_{2}(k)}{m_{12}(k) x_{2}(k)+x_{1}(k)}\right\} \\
x_{2}(k+1)=x_{2}(k) \exp \left\{-a_{2}(k)+\frac{a_{21}(k) x_{1}\left(k-\tau_{2}(k)\right)}{m_{12}(k) x_{2}\left(k-\tau_{2}(k)\right)+x_{1}\left(k-\tau_{2}(k)\right)}-\frac{a_{23}(k) x_{3}(k)}{m_{23}(k) x_{3}(k)+x_{2}(k)}\right\} \\
x_{3}(k+1)=x_{3}(k) \exp \left\{-a_{3}(k)+\frac{a_{32}(k) x_{2}\left(k-\tau_{3}(k)\right)}{m_{23}(k) x_{3}\left(k-\tau_{3}(k)\right)+x_{2}\left(k-\tau_{3}(k)\right)}\right\}
\end{array}\right.
$$

where $k=0,1,2, \ldots$ and all the variables and parameters have the same biological meanings as those in system (1).

The main task of this article is to discuss the dynamics of system (3). That is, applying Mawhin's continuous theorem [21] to study the existence of positive periodic solutions of (3) and investigating the global asymptotical stability of system (3) by means of the method of Lyapunov function. The main innovation point lies in better applying computer simulation to explain the changing law of biological population.

The remainder of the paper is organized as follows. In Section 2, a easily verifiable sufficient condition for the existence of positive solutions of difference equations is obtained by the continuation theorem and priori estimations. The sufficient condition for the global asymptotical stability of system (3) when all the delays are zero is presented in Section 3. In Section 4, we give some computer simulations.

\section{Existence of Positive Periodic Solutions}

For convenience and simplicity in the following discussion, we always use the following notations throughout the paper:

$$
\begin{aligned}
I_{\omega} & :=\{0,1,2, \ldots, \omega-1\}, \\
\bar{f} & :=\frac{1}{\omega} \sum_{k=0}^{\omega-1} f(k), \\
f^{L} & :=\min _{k \in Z}\{f(k)\}, \\
f_{M} & :=\max _{k \in Z}\{f(k)\},
\end{aligned}
$$


where $f(k)$ is an $\omega$-periodic sequence of real numbers defined for $k \in Z$. Let $Z$ denote the integer number, $R$ denote the real number, $R^{+}$denote the nonnegative real number, and $R^{3}$ denote the three-dimensional real vector.

We always assume that

(H1) $a_{i}(i=1,2,3), a_{11}, a_{12}, a_{21}, a_{23}, a_{32}, m_{12}, m_{23}$ :

$Z \longrightarrow R^{+}$are $\omega$ periodic, i.e.,

$$
\begin{aligned}
a_{i}(k+\omega) & =a_{i}(k)(i=1,2,3), \\
a_{11}(k+\omega) & =a_{11}(k), \\
a_{12}(k+\omega) & =a_{12}(k), \\
a_{21}(k+\omega) & =a_{21}(k), \\
a_{23}(k+\omega) & =a_{23}(k), \\
m_{12}(k+\omega) & =m_{12}(k), \\
m_{23}(k+\omega) & =m_{23}(k), \quad \text { for any } k \in Z .
\end{aligned}
$$

In order to explore the existence of positive periodic solutions of (3) and for the reader's convenience, we shall first introduce a few concepts and results without proof, borrowing from Gaines and Mawhin [21].

Let $X$ and $Y$ be normed vector spaces, $L: \operatorname{Dom} L \subset X \longrightarrow Y$ is a linear mapping, and $N: X \longrightarrow Y$ is a continuous mapping. The mapping $L$ will be called a Fredholm mapping of index zero if $\operatorname{dimKer} L=$ codim $\operatorname{Im} L<+\infty$ and $\operatorname{Im} L$ is closed in $Y$. If $L$ is a Fredholm mapping of index zero and there exist continuous projectors $P: X \longrightarrow X$ and $Q: Y \longrightarrow Y$ such that $\operatorname{Im} P=$ $\operatorname{Ker} L$ and $\operatorname{Im} L=\operatorname{Ker} Q=\operatorname{Im}(I-Q)$, It follows that $L \mid \operatorname{Dom} L \cap \operatorname{Ker} P:(I-P) X \longrightarrow \operatorname{Im} L$ is invertible. We denote the inverse of that map by $K_{P}$. If $\Omega$ is an open-bounded subset of $X$, the mapping $N$ will be called $L$-compact on $\bar{\Omega}$ if $Q N(\bar{\Omega})$ is bounded and $K_{P}(I-Q) N: \bar{\Omega} \longrightarrow X$ is compact. Since $\operatorname{Im} Q$ is isomorphic to $\operatorname{Ker} L$, there exist isomorphisms $J: \operatorname{Im} Q \longrightarrow \operatorname{Ker} L$.

Lemma 1 (see [21], continuation theorem). Let $L$ be a Fredholm mapping of index zero, and let $N$ be $L-$ compact on $\bar{\Omega}$. Suppose

(a) For each $\lambda \in(0,1)$, every solution $x$ of $L x=\lambda N x$ is such that $x \notin \partial \Omega$

(b) $Q N x \neq 0$ for each $x \in \operatorname{Ker} L \cap \partial \Omega$, and $\operatorname{deg}\{J Q N$, $\Omega \cap \operatorname{Ker} L, 0\} \neq 0$

Then, the equation $L x=N x$ has at least one solution lying in Dom $L \cap \bar{\Omega}$.

Lemma 2 (see [20]). Let $g: Z \longrightarrow R$ be $\omega$ periodic, i.e., $g(k+\omega)=g(k)$; then, for any fixed $k_{1}, k_{2} \in I_{\omega}$ and any $k \in Z$, one has

$$
\begin{aligned}
& g(k) \leq g\left(k_{1}\right)+\sum_{s=0}^{\omega-1}|g(s+1)-g(s)|, \\
& g(k) \geq g\left(k_{2}\right)-\sum_{s=0}^{\omega-1}|g(s+1)-g(s)| .
\end{aligned}
$$

Lemma 3. Assume that $\bar{a}_{2}<\bar{a}_{21}$ and $\bar{a}_{3}<\bar{a}_{32}$; then, the system algebraic equations

$$
\left\{\begin{array}{l}
\bar{a}_{1}-\bar{a}_{11} v_{1}=0, \\
\bar{a}_{2}-\frac{1}{\omega} \sum_{k=0}^{\omega-1} \frac{a_{21}(k) v_{1}}{m_{12}(k) v_{2}+v_{1}}=0, \\
\bar{a}_{3}-\frac{1}{\omega} \sum_{k=0}^{\omega-1} \frac{a_{32}(k) v_{2}}{m_{23}(k) v_{3}+v_{2}}=0,
\end{array}\right.
$$

have a unique solution $\left(v_{1}^{*}, v_{2}^{*}, v_{3}^{*}\right)$ with $v_{i}^{*}>0, \quad i=1,2,3$.

Proof. Obviously, $v_{1}=\bar{a}_{1} / \bar{a}_{11}>0$. Substituting $v_{1}=\bar{a}_{1} / \bar{a}_{11}$ into the second equation of system (7) and simplifying, we obtain

$$
\bar{a}_{2}-\frac{1}{\omega} \sum_{k=0}^{\omega-1} \frac{a_{21}(k)}{\left(m_{12}(k) \bar{a}_{11} / \bar{a}_{1}\right) v_{2}+1}=0 .
$$

In the following, we define the function:

$$
f(\theta)=\bar{a}_{2}-\frac{1}{\omega} \sum_{k=0}^{\omega-1} \frac{a_{21}(k)}{\left(m_{12}(k) \bar{a}_{11} / \bar{a}_{1}\right) \theta+1}, \quad \theta \geq 0 .
$$

It is easy to see that

$$
\begin{gathered}
f(0)=\bar{a}_{2}-\frac{1}{\omega} \sum_{k=0}^{\omega-1} a_{21}(k)=\bar{a}_{2}-\bar{a}_{21}<0, \\
\lim _{\theta \longrightarrow+\infty} f(\theta)=\bar{a}_{2}>0 .
\end{gathered}
$$

Then, it follows from the zero-point theorem and monotonicity of $f(\theta)$ that there exists a unique $v_{2}^{*}>0$ such that $f\left(v_{2}^{*}\right)=0$. Similarly, substituting $v_{2}^{*}$ into the third equation of system (7) and simplifying, we have

$$
\bar{a}_{3}-\frac{1}{\omega} \sum_{k=0}^{\omega-1} \frac{a_{32}(k)}{\left(m_{23}(k) / v_{2}^{*}\right) v_{3}+1}=0 .
$$

We define the function

$$
g(\gamma)=\bar{a}_{3}-\frac{1}{\omega} \sum_{k=0}^{\omega-1} \frac{a_{32}(k)}{\left(m_{23}(k) / v_{2}^{*}\right) \gamma+1}, \quad \gamma \geq 0 .
$$

Clearly, 


$$
\begin{gathered}
g(0)=\bar{a}_{3}-\frac{1}{\omega} \sum_{k=0}^{\omega-1} a_{32}(k)=\bar{a}_{3}-\bar{a}_{32}<0, \\
\lim _{\theta \longrightarrow+\infty} g(\gamma)=\bar{a}_{3}>0 .
\end{gathered}
$$

Then, it follows from the zero-point theorem and monotonicity of $g(\gamma)$ that there exists a unique $v_{3}^{*}>0$ such that $g\left(v_{3}^{*}\right)=0$. The proof is complete.

Define

$$
l_{3}=\left\{z=\{z(k)\}: z(k)=\left(z_{1}(k), z_{2}(k), z_{3}(k)\right)^{T} \in R^{3}, k \in Z\right\} \text {. }
$$

Let $l^{\omega} \subset l_{3}$ denote the subspace of all $\omega$ periodic sequences equipped with the usual supremum norm $\|\cdot\|$, i.e., $\|z\|=\left|z_{1}(k)\right|+\left|z_{2}(k)\right|+\left|z_{3}(k)\right|$ for any $z=\{z(k): k \in Z\}$ $\in l^{\omega}$. It is easy to show that $l^{\omega}$ is a finite-dimensional Banach space.

$$
\begin{aligned}
& \text { Let } \\
& \qquad \begin{array}{l}
l_{0}^{\omega}=\left\{z=\{z(k)\} \in l^{\omega}: \sum_{k=0}^{\omega-1} z(k)=0\right\}, \\
l_{c}^{\omega}=\left\{z=\{z(k)\} \in l^{\omega}: z(k)=h \in R^{3}, k \in Z\right\} .
\end{array}
\end{aligned}
$$

Then, it follows that $l_{0}^{\omega}$ and $l_{c}^{\omega}$ are both closed linear subspaces of $l^{\omega}$ and

$$
l^{\omega}=l_{0}^{\omega}+l_{c}^{\omega}, \operatorname{dim} l_{c}^{\omega}=3 .
$$
result.

In the following, we will ready to establish our

Theorem 1. Let $S_{1}$ be defined by (37). Under condition (H1), suppose that the following conditions

$$
\begin{gathered}
(\mathrm{H} 2) \bar{a}_{1}>\left(\frac{\overline{a_{12}}}{m_{12}}\right), \\
\bar{a}_{1} \exp \left(-S_{1}\right)>\bar{a}_{11}, \\
(\mathrm{H} 3) \bar{a}_{2}<\bar{a}_{21}, \bar{a}_{3}<\bar{a}_{32},
\end{gathered}
$$

hold; then, system (3) has at least an $\omega$ positive periodic solution.

Proof. First, we make the change of variables $x_{i}(t)=$ $\exp \left(u_{i}(t)\right)(i=1,2,3)$; then, (3) can be reformulated as

$$
\left\{\begin{array}{l}
u_{1}(k+1)-u_{1}(k)=f_{1}\left(u_{1}, u_{2}, u_{3}\right), \\
u_{2}(k+1)-u_{2}(k)=f_{2}\left(u_{1}, u_{2}, u_{3}\right), \\
u_{3}(k+1)-u_{3}(k)=f_{3}\left(u_{1}, u_{2}, u_{3}\right)
\end{array}\right.
$$

where

$$
\begin{aligned}
f_{1}\left(u_{1}, u_{2}, u_{3}\right)= & a_{1}(k)-a_{11}(k) \exp \left(u_{1}\left(k-\tau_{1}(k)\right)\right)-\frac{a_{12}(k) \exp \left(u_{2}(k)\right)}{m_{12}(k) \exp \left(u_{2}(k)\right)+\exp \left(u_{1}(k)\right)} \\
f_{2}\left(u_{1}, u_{2}, u_{3}\right)= & -a_{2}(k)+\frac{a_{21}(k) \exp \left(u_{1}\left(k-\tau_{2}(k)\right)\right)}{m_{12}(k) \exp \left(u_{2}\left(k-\tau_{2}(k)\right)\right)+\exp \left(u_{1}\left(k-\tau_{2}(k)\right)\right)} \\
& -\frac{a_{23}(k) \exp \left(u_{3}(k)\right)}{m_{23}(k) \exp \left(u_{3}(k)\right)+\exp \left(u_{2}(k)\right)} \\
f_{3}\left(u_{1}, u_{2}, u_{3}\right)= & -a_{3}(k)+\frac{a_{32}(k) \exp \left(u_{2}\left(k-\tau_{3}(k)\right)\right)}{m_{23}(k) \exp \left(u_{3}\left(k-\tau_{3}(k)\right)\right)+\exp \left(u_{2}\left(k-\tau_{3}(k)\right)\right)} .
\end{aligned}
$$

Let $X=Y=l^{\omega}$,

$$
\begin{aligned}
& (L u)(k)=u(k+1)-u(k) \\
& (N u)(k)=\left(\begin{array}{l}
f_{1}\left(u_{1}, u_{2}, u_{3}\right) \\
f_{2}\left(u_{1}, u_{2}, u_{3}\right) \\
f_{3}\left(u_{1}, u_{2}, u_{3}\right)
\end{array}\right),
\end{aligned}
$$

where $u \in X, k \in Z$. Then, it is trivial to see that $L$ is a bounded linear operator and

$$
\operatorname{Ker} L=l_{c}^{\omega}, \quad \operatorname{Im} L=l_{0}^{\omega},
$$

$$
\operatorname{dim} \operatorname{Ker} L=3=\operatorname{codim} \operatorname{Im} L
$$

Then, it follows that $L$ is a Fredholm mapping of index zero. Define

$$
\begin{aligned}
& P u=\frac{1}{\omega} \sum_{s=0}^{\omega-1} u(s), \quad u \in X, \\
& Q z=\frac{1}{\omega} \sum_{s=0}^{\omega-1} z(s), \quad z \in Y .
\end{aligned}
$$

It is not difficult to show that $P$ and $Q$ are continuous projectors such that

$$
\begin{aligned}
& \operatorname{Im} P=\operatorname{Ker} L, \\
& \operatorname{Im} L=\operatorname{Ker} Q=\operatorname{Im}(I-Q) .
\end{aligned}
$$


Furthermore, the generalized inverse (to $L$ ) $k_{P}$ : $\operatorname{Im} L \longrightarrow \operatorname{Ker} P \cap \operatorname{Dom} L$ exists and is given by

$$
K_{P}(z)=\sum_{s=0}^{\omega-1} z(s)-\frac{1}{\omega} \sum_{s=0}^{\omega-1}(\omega-s) z(s) .
$$

Obviously, $Q N$ and $K_{P}(I-Q) N$ are continuous. Since $X$ is a finite-dimensional Banach space, using the Ascoli-Arzela theorem, it is not difficult to show that $\overline{K_{P}(I-Q) N(\bar{\Omega})}$ is compact for any open-bounded set $\Omega \subset X$. Moreover, $Q N(\bar{\Omega})$ is bounded. Thus, $N$ is $L$-compact on $\bar{\Omega}$ with any open-bounded set $\Omega \subset X$.
Now, we are at the point to search for an appropriate open-bounded subset $\Omega$ for the application of the continuation theorem. Corresponding to the operator equation $L u=\lambda N u, \lambda \in(0,1)$, we have

$$
\left\{\begin{array}{l}
u_{1}(k+1)-u_{1}(k)=\lambda f_{1}\left(u_{1}, u_{2}, u_{3}\right), \\
u_{2}(k+1)-u_{2}(k)=\lambda f_{2}\left(u_{1}, u_{2}, u_{3}\right), \\
u_{3}(k+1)-u_{3}(k)=\lambda f_{3}\left(u_{1}, u_{2}, u_{3}\right) .
\end{array}\right.
$$

Suppose that $u(k)=\left(u_{1}(k), u_{2}(k), u_{3}(k)\right)^{T} \in X$ is an arbitrary solution of system (28) for a certain $\lambda \in(0,1)$; summing both sides of (28) from 0 to $\omega-1$ with respect to $k$, respectively, we obtain

$$
\left\{\begin{array}{l}
\sum_{k=0}^{\omega-1}\left[a_{11}(k) \exp \left(u_{1}\left(k-\tau_{1}(k)\right)\right)+\frac{a_{12}(k) \exp \left(u_{2}(k)\right)}{m_{12}(k) \exp \left(u_{2}(k)\right)+\exp \left(u_{1}(k)\right)}\right]=\bar{a}_{1} \omega, \\
\sum_{k=0}^{\omega-1}\left[\frac{a_{21}(k) \exp \left(u_{1}\left(k-\tau_{2}(k)\right)\right)}{m_{12}(k) \exp \left(u_{2}\left(k-\tau_{2}(k)\right)\right)+\exp \left(u_{1}\left(k-\tau_{2}(k)\right)\right)}-\frac{a_{23}(k) \exp \left(u_{3}(k)\right)}{m_{23}(k) \exp \left(u_{3}(k)\right)+\exp \left(u_{2}(k)\right)}\right]=\bar{a}_{2} \omega, \\
\sum_{k=0}^{\omega-1}\left[\frac{a_{32}(k) \exp \left(u_{2}\left(k-\tau_{3}(k)\right)\right)}{m_{23}(k) \exp \left(u_{3}\left(k-\tau_{3}(k)\right)\right)+\exp \left(u_{2}\left(k-\tau_{3}(k)\right)\right)}\right]=\bar{a}_{3} \omega .
\end{array}\right.
$$

In view of the hypothesis that $u=\{u(k)\} \in X$, there exist

It follows from (28) and (29) that $\xi_{i}, \eta_{i} \in I_{\omega}(i=1,2,3)$ such that

$$
\begin{aligned}
& u_{i}\left(\xi_{i}\right)=\min _{k \in I_{\omega}}\left\{u_{i}(k)\right\}, \\
& u_{i}\left(\eta_{i}\right)=\max _{k \in I_{\omega}}\left\{u_{i}(k)\right\} .
\end{aligned}
$$

$$
\begin{aligned}
\sum_{k=0}^{\omega-1}\left|u_{1}(k+1)-u_{1}(k)\right| & \leq \lambda\left|\sum_{k=0}^{\omega-1} a_{1}(k)-a_{11}(k) \exp \left(u_{1}\left(k-\tau_{1}(k)\right)\right)-\frac{a_{12}(k) \exp \left(u_{2}(k)\right)}{m_{12}(k) \exp \left(u_{2}(k)\right)+\exp \left(u_{1}(k)\right)}\right| \\
& <2 \bar{a}_{1} \omega,
\end{aligned}
$$

$$
\begin{aligned}
\sum_{k=0}^{\omega-1}\left|u_{2}(k+1)-u_{2}(k)\right| & \leq \lambda \mid \sum_{k=0}^{\omega-1}\left[-a_{2}(k)+\frac{a_{21}(k) \exp \left(u_{1}\left(k-\tau_{2}(k)\right)\right)}{m_{12}(k) \exp \left(u_{2}\left(k-\tau_{2}(k)\right)\right)+\exp \left(u_{1}\left(k-\tau_{2}(k)\right)\right)}-\frac{a_{23}(k) u_{3}(k)}{m_{23}(k) \exp \left(u_{3}(k)\right)+\exp \left(u_{2}(k)\right)} \mid\right. \\
& <2 \bar{a}_{2} \omega,
\end{aligned}
$$

$$
\sum_{k=0}^{\omega-1}\left|u_{3}(k+1)-u_{3}(k)\right| \leq \lambda\left|\sum_{k=0}^{\omega-1}\left[-a_{3}(k)+\frac{a_{32}(k) \exp \left(u_{2}\left(k-\tau_{3}(k)\right)\right)}{m_{23}(k) \exp \left(u_{3}\left(k-\tau_{3}(k)\right)\right)+\exp \left(u_{2}\left(k-\tau_{3}(k)\right)\right)}\right]\right|<2 \bar{a}_{3} \omega .
$$


By the first equation of (29), we have

$$
\begin{aligned}
& \sum_{k=0}^{\omega-1}\left[a_{11}(k) \exp \left(u_{1}\left(k-\tau_{1}(k)\right)\right)\right] \\
& \quad<\bar{a}_{1} \omega, \sum_{k=0}^{\omega-1}\left[a_{11}(k) \exp \left(u_{1}\left(k-\tau_{1}(k)\right)\right)+\frac{a_{12}(k)}{m_{12}(k)}\right] \geq \bar{a}_{1} \omega,
\end{aligned}
$$

which leads to

$$
u_{1}\left(\xi_{1}\right)<\ln \left[\frac{\bar{a}_{1}}{\bar{a}_{11}}\right], u_{1}\left(\eta_{1}\right)>\ln \left[\frac{\bar{a}_{1}-\left(\overline{a_{12} / m_{12}}\right)}{\bar{a}_{11}}\right] .
$$

By (31) and (35) and Lemma 2, we obtain

$$
\begin{aligned}
u_{1}(k) & \leq u\left(\xi_{1}\right)+\sum_{s=0}^{\omega-1}\left|u_{1}(s+1)-u_{1}(s)\right| \\
& \leq \ln \left[\frac{\bar{a}_{1}}{\bar{a}_{11}}\right]+2 \bar{a}_{1} \omega:=M_{1}, \\
u_{1}(k) & \geq u_{1}\left(\eta_{1}\right)-\sum_{s=0}^{\omega-1}\left|u_{1}(s+1)-u_{1}(s)\right| \\
& \geq \ln \left[\frac{\bar{a}_{1}-\left(\overline{a_{12} / m_{12}}\right)}{\bar{a}_{11}}\right]-2 \bar{a}_{1} \omega:=m_{1} .
\end{aligned}
$$

Thus,

$$
\max _{k \in I_{\omega}}\left\{u_{1}(k)\right\}<\max \left\{\left|m_{1}\right|,\left|M_{1}\right|\right\}:=S_{1} .
$$

In view of the second equation of (29) and (37), it is easy to obtain

$$
\sum_{k=0}^{\omega-1}\left[\frac{a_{21}(k) \exp \left(u_{1}\left(k-\tau_{2}(k)\right)\right)}{m_{12}(k) \exp \left(u_{2}\left(k-\tau_{2}(k)\right)\right)}\right]>\bar{a}_{2} \omega
$$

then

$$
\sum_{k=0}^{\omega-1}\left[\frac{a_{21}(k) \exp \left(S_{1}\right)}{m_{12}(k) \exp \left(u_{2}\left(\xi_{2}\right)\right)}\right]>\bar{a}_{2} \omega
$$

Thus,

$$
u_{2}\left(\xi_{2}\right)<\ln \left[\frac{\left(\overline{a_{21} / m_{12}}\right) \exp \left(S_{1}\right)}{\bar{a}_{2}}\right] \text {. }
$$

From the first equation of (29) and (37), we obtain $\sum_{k=0}^{\omega-1}\left[a_{11}(k) \exp \left(S_{1}\right)+\frac{a_{12}(k) \exp \left(u_{2}\left(\eta_{2}\right)\right)}{\exp \left(-S_{1}\right)}\right]>\bar{a}_{1} \omega$

Then,

$$
u_{2}\left(\eta_{2}\right)>\ln \left[\frac{\bar{a}_{1} \exp \left(-S_{1}\right)-\bar{a}_{11}}{\bar{a}_{12}}\right] .
$$

It follows from (40) and (42) and Lemma 2 that

$$
\begin{aligned}
u_{2}(k) & \leq u\left(\xi_{2}\right)+\sum_{s=0}^{\omega-1}\left|u_{2}(s+1)-u_{2}(s)\right| \\
& \leq \ln \left[\frac{\left(\overline{a_{21} / m_{12}}\right) \exp \left(S_{1}\right)}{\bar{a}_{2}}\right]+2 \bar{a}_{2} \omega:=M_{2}, \\
u_{2}(k) & \geq u_{2}\left(\eta_{2}\right)-\sum_{s=0}^{\omega-1}\left|u_{2}(s+1)-u_{2}(s)\right| \\
& \geq \ln \left[\frac{\bar{a}_{1} \exp \left(-S_{1}\right)-\bar{a}_{11}}{\bar{a}_{12}}\right]-2 \bar{a}_{2} \omega:=m_{2} .
\end{aligned}
$$

Thus,

$$
\max _{k \in I_{\omega}}\left\{u_{2}(k)\right\}<\max \left\{\left|m_{2}\right|,\left|M_{2}\right|\right\}:=S_{2} .
$$

By the third equation of (29), we obtain

$$
\sum_{k=0}^{\omega-1}\left[\frac{a_{32}(k) \exp \left(u_{2}\left(k-\tau_{3}(k)\right)\right)}{m_{23}(k) \exp \left(u_{3}\left(k-\tau_{3}(k)\right)\right)}\right]>\bar{a}_{3} \omega,
$$

which leads to

$$
\sum_{k=0}^{\omega-1}\left[\frac{a_{32}(k) \exp \left(S_{2}\right)}{m_{23}(k) \exp \left(u_{3}\left(\xi_{3}\right)\right)}\right]>\bar{a}_{3} \omega
$$

Then,

$$
u_{3}\left(\xi_{3}\right)<\ln \left[\frac{\left(\overline{a_{32} / m_{23}}\right) \exp \left(S_{2}\right)}{\bar{a}_{3}}\right] .
$$

By the third equation of (29), we also obtain

$$
\sum_{k=0}^{\omega-1}\left[\frac{a_{32}(k) \exp \left(-S_{2}\right)}{m_{23}^{M} \exp \left(u_{3}\left(\eta_{3}\right)\right)+\exp \left(-S_{2}\right)}\right]<\bar{a}_{3} \omega .
$$

Thus, we obtain 


$$
u_{3}\left(\eta_{3}\right)>\ln \left[\frac{\left(\bar{a}_{32}-\bar{a}_{3}\right) \exp \left(-S_{2}\right)}{\bar{a}_{3} m_{23}^{M}}\right] \text {. }
$$

From (48) and (50) and Lemma 2, we derive

$$
\begin{aligned}
u_{3}(k) & \leq u\left(\xi_{3}\right)+\sum_{s=0}^{\omega-1}\left|u_{3}(s+1)-u_{3}(s)\right| \\
& \leq \ln \left[\frac{\left(\overline{a_{32} / m_{23}}\right) \exp \left(S_{2}\right)}{\bar{a}_{3}}\right]+2 \bar{a}_{3} \omega:=M_{3}, \\
u_{3}(k) & \geq u_{3}\left(\eta_{3}\right)-\sum_{s=0}^{\omega-1}\left|u_{2}(s+1)-u_{2}(s)\right| \\
& \geq \ln \left[\frac{\left(\bar{a}_{32}-\bar{a}_{3}\right) \exp \left(-S_{2}\right)}{\bar{a}_{3} m_{23}^{M}}\right]-2 \bar{a}_{3} \omega:=m_{3} .
\end{aligned}
$$

$$
Q N y=\left(\begin{array}{c}
\bar{a}_{1}-\bar{a}_{11} \exp \left(u_{1}\right)-\frac{1}{\omega} \sum_{k=0}^{\omega-1} \frac{a_{21}(k) \exp \left(u_{1}\right)}{m_{12}(k) \exp \left(u_{2}\right)+\exp \left(u_{1}\right)} \\
-\bar{a}_{2}+\frac{1}{\omega} \sum_{k=0}^{\omega-1} \frac{a_{21}(k) \exp \left(u_{1}\right)}{m_{12}(k) \exp \left(u_{2}\right)+\exp \left(u_{1}\right)}-\frac{1}{\omega} \sum_{k=0}^{\omega-1} \frac{a_{23}(k) \exp \left(u_{3}\right)}{m_{23}(k) \exp \left(u_{3}\right)+\exp \left(u_{3}\right)} \\
-\bar{a}_{3}+\frac{1}{\omega} \sum_{k=0}^{\omega-1} \frac{a_{32}(k) \exp \left(u_{2}\right)}{m_{23}(k) \exp \left(u_{3}\right)+\exp \left(u_{2}\right)}
\end{array}\right) \neq 0 .
$$

Now, let us consider homotopic $\phi\left(u_{1}, u_{2}, u_{3}\right.$, $\mu)=\mu Q N u+(1-\mu) G u, \mu \in[0,1]$, where

$$
G z=\frac{1}{\omega} \sum_{k=0}^{\omega-1}\left(\begin{array}{c}
\bar{a}_{1}-\bar{a}_{11} \exp \left(u_{1}\right) \\
-\bar{a}_{2}+\frac{1}{\omega} \sum_{k=0}^{\omega-1} \frac{a_{21}(k) \exp \left(u_{1}\right)}{m_{12}(k) \exp \left(u_{2}\right)+\exp \left(u_{1}\right)} \\
-\bar{a}_{3}+\frac{1}{\omega} \sum_{k=0}^{\omega-1} \frac{a_{32}(k) \exp \left(u_{2}\right)}{m_{23}(k) \exp \left(u_{3}\right)+\exp \left(u_{2}\right)}
\end{array}\right) .
$$

In fact, when $\phi\left(u_{1}, u_{2}, u_{3}, 0\right)=G u$ and $\phi\left(u_{1}, u_{2}, u_{3}\right.$, $1)=Q N u$, then $\phi$ is a homptopic mapping. Letting $J$ be the identity mapping and by direct calculation, we obtain

$$
\begin{aligned}
& \operatorname{deg}\left\{\operatorname{JQN}\left(u_{1}, u_{2}, u_{3}\right)^{T} ; \Omega \cap \operatorname{ker} L ; 0\right\} \\
& =\operatorname{deg}\left\{Q N\left(u_{1}, u_{2}, u_{3}\right)^{T} ; \Omega \cap \operatorname{ker} L ; 0\right\} \\
& =\operatorname{deg}\left\{\phi\left(u_{1}, u_{2}, u_{3}, 1\right) ; \Omega \cap \operatorname{ker} L ; 0\right\} \\
& =\operatorname{deg}\left\{\phi\left(u_{1}, u_{2}, u_{3}, 0\right) ; \Omega \cap \operatorname{ker} L ; 0\right\} \\
& =\operatorname{sign}\left\{\operatorname{det}\left(\begin{array}{ccc}
\chi_{11} & 0 & 0 \\
\chi_{21} & \chi_{22} & 0 \\
0 & \chi_{32} & \chi_{33}
\end{array}\right)\right\},
\end{aligned}
$$

Thus,

$$
\max _{k \in I_{\omega}}\left\{u_{3}(k)\right\}<\max \left\{\left|m_{3}\right|,\left|M_{3}\right|\right\}:=S_{3}
$$

Obviously, $S_{i}(i=1,2,3)$ are independent of the choice of $\lambda \in(0,1)$. Take $S=\max \left\{S_{1}, S_{2}, S_{3}\right\}+S_{0}$, where $S_{0}$ is taken sufficiently large such that $\left|\ln v_{1}^{*}\right|+\left|\ln v_{2}^{*}\right|+\left|\ln v_{3}^{*}\right|<S_{0}$, where $\left(v_{1}^{*}, v_{2}^{*}, v_{3}^{*}\right)^{T}$ is the unique positive solution of (7).

Now, we have proved that any solution $u=\{u(k)\}=$ $\left\{\left(u_{1}(k), u_{2}(k), u_{3}(k)\right)^{T}\right\}$ of $(28)$ in $X$ satisfies $\|u\|<S, k \in Z$.

Let $\Omega:=\{u=\{u(k)\} \in X:\|u\|<S\}$; then, it is easy to see that $\Omega$ is an open-bounded set in $X$ and verifies requirement (a) of Lemma 2. When $u \in \partial \Omega \cap \operatorname{Ker} L, u=\left\{\left(u_{1}, u_{2}, u_{3}\right)^{T}\right\}$ is a constant vector in $R^{3}$ with $\|u\|=\left|u_{1}\right|+\left|u_{2}\right|+\left|u_{3}\right|=S$. Then, 


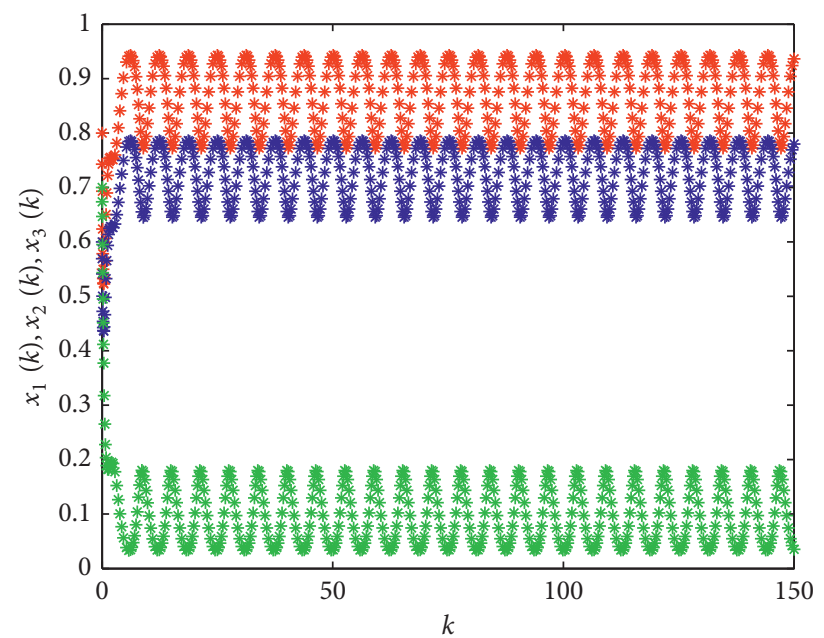

FiguRE 1: The time histories of $t-x_{1}, t-x_{2}$, and $t-x_{3}$, where the red line represents $x_{1}$, the blue line represents $x_{2}$, and the green line represents $x_{3}$.

periodic solution in $\operatorname{Dom} L \cap \bar{\Omega}$, say $u^{*}=\left\{u^{*}(k)\right\}=$ $\left\{\left(u_{1}^{*}(k), u_{2}^{*}(k), u_{3}^{*}(k)\right)^{T}\right.$; then, it follows that $\left(\exp \left\{u_{1}^{*}\right.\right.$ $(k)\}, \exp \left\{u_{2}^{*}(k), \exp \left\{u_{3}^{*}(k)\right\}\right)^{T}$ is an $\omega$ periodic solution of system (3) with strictly positive components. The proof is complete.

\section{Global Asymptotic Stability}

In this section, we will present sufficient conditions for the globally asymptotical stability of system (3) when all the delays are zero.
Theorem 2. Let $A_{1}^{*}, A_{2}^{*}$, and $A_{3}^{*}$ are defined by (67), (68), and (69), respectively. Assume that (H1)-(H3) are satisfied. Furthermore, suppose that there exist positive constants $\eta, \delta_{1}, \delta_{2}$, and $\delta_{3}$ such that $A_{i}^{*}>0(i=1,2,3)$ and

(i) $1-a_{11}(k) \exp \left(M_{1}\right)-\frac{a_{12}(k) \exp \left(M_{2}\right)}{m_{12}(k) \exp \left(m_{2}\right)+\exp \left(m_{1}\right)}+\frac{a_{12}(k) \exp \left(m_{1}+m_{2}\right)}{\left(m_{12}(k) \exp \left(M_{2}\right)+\exp \left(M_{1}\right)\right)^{2}} \geq \eta$,

(ii) $\frac{\exp \left(m_{2}\right)-a_{21}(k) \exp \left(M_{1}+M_{2}\right)-a_{23}(k) \exp \left(M_{2}+M_{3}\right)}{m_{12}(k) \exp \left(M_{2}\right)+\exp \left(M_{1}\right)}-\frac{a_{21}(k) a_{23}(k) \exp \left(M_{1}+M_{2}+M_{3}\right)}{\left(m_{12}(k) \exp \left(m_{2}\right)+\exp \left(m_{1}\right)\right)^{2}} \geq \eta$,

(iii) $\frac{m_{23}(k)}{m_{23}(k) \exp \left(M_{3}\right)+\exp \left(M_{2}\right)} \leq 1$.

Then, the positive $\omega$-periodic solution of system (3) is globally asymptotically stable.

Proof. In view of Theorem 2, there exists a positive periodic solution $\left\{x_{1}^{*}(k), x_{2}^{*}(k), x_{3}^{*}(k)\right\}$ of system (3). We prove below that it is uniformly asymptotically stable. First, we introduce the change of variables as follows:

$$
\begin{aligned}
N_{1}(k) & =x_{1}(k)-x_{1}^{*}(k), N_{2}(k)=x_{2}(k)-x_{2}^{*}(k), N_{3}(k) \\
& =x_{3}(k)-x_{3}^{*}(k) .
\end{aligned}
$$

Then, it follows from (3) that 


$$
\begin{aligned}
& N_{1}(k+1)=x_{1}(k+1)-x_{1}^{*}(k+1) \\
& =x_{1}(k) \exp \left\{a_{1}(k)-a_{11}(k) x_{1}(k)-\frac{a_{12}(k) x_{2}(k)}{m_{12}(k) x_{2}(k)+x_{1}(k)}\right\} \text {, } \\
& -x_{1}^{*}(k) \exp \left\{a_{1}(k)-a_{11}(k) x_{1}^{*}(k)-\frac{a_{12}(k) x_{2}^{*}(k)}{m_{12}(k) x_{2}^{*}(k)+x_{1}^{*}(k)}\right\} \\
& =\left\{x _ { 1 } ( k ) \operatorname { e x p } \left[-a_{11}(k) N_{1}(k)-a_{12}(k)\left(\frac{x_{2}(k)}{m_{12}(k) x_{2}(k)+x_{1}(k)}\right.\right.\right. \\
& \left.\left.\left.-\frac{x_{2}^{*}(k)}{m_{12}(k) x_{2}^{*}(k)+x_{1}^{*}(k)}\right)\right]-x_{1}^{*}(k)\right\} \frac{x_{1}^{*}(k+1)}{x_{1}^{*}(k)}, \\
& =\left\{\begin{array}{c}
{\left[1-a_{11}(k) x_{1}^{*}(k)-\frac{a_{12}(k) x_{2}^{*}(k)}{m_{12}(k) x_{2}^{*}(k)+x_{1}^{*}(k)}+\frac{a_{12}(k) x_{1}^{*}(k) x_{2}^{*}(k)}{\left(m_{12}(k) x_{2}^{*}(k)+x_{1}^{*}(k)\right)^{2}}\right] \frac{N_{1}(k)}{x_{1}^{*}(k)}} \\
-\frac{a_{12}(k)}{m_{12}(k) x_{2}^{*}(k)+x_{1}^{*}(k)} N_{2}(k)+\rho_{1}
\end{array}\right\} x_{1}^{*}(k+1), \\
& N_{2}(k+1)=x_{2}(k+1)-x_{2}^{*}(k+1) \\
& =x_{2}(k) \exp \left\{-a_{2}(k)+\frac{a_{21}(k) x_{1}(k)}{m_{12}(k) x_{2}(k)+x_{1}(k)}-\frac{a_{23}(k) x_{3}(k)}{m_{23}(k) x_{3}(k)+x_{2}(k)}\right\} \\
& -x_{2}^{*}(k) \exp \left\{-a_{2}(k)+\frac{a_{21}(k) x_{1}^{*}(k)}{m_{12}(k) x_{2}^{*}(k)+x_{1}^{*}(k)}-\frac{a_{23}(k) x_{3}^{*}(k)}{m_{23}(k) x_{3}^{*}(k)+x_{2}^{*}(k)}\right\} \\
& =\left[x _ { 2 } ( k ) \operatorname { e x p } \left\{\left(\frac{a_{21}(k) x_{1}(k)}{m_{12}(k) x_{2}(k)+x_{1}(k)}-\frac{a_{21}(k) x_{1}^{*}(k)}{m_{12}(k) x_{2}^{*}(k)+x_{1}^{*}(k)}\right)\right.\right. \\
& \left.\left.-\left(\frac{a_{23}(k) x_{3}(k)}{m_{23}(k) x_{3}(k)+x_{2}(k)}-\frac{a_{23}(k) x_{3}^{*}(k)}{m_{23}(k) x_{3}^{*}(k)+x_{2}^{*}(k)}\right)\right\}-x_{2}^{*}(k)\right] \frac{x_{2}^{*}(k+1)}{x_{2}^{*}(k)} \\
& =\left\{\left[\frac{a_{21}(k)}{m_{12}(k) x_{2}^{*}(k)+x_{1}^{*}(k)}-\frac{a_{21}(k) x_{1}^{*}(k)}{\left(m_{12}(k) x_{2}^{*}(k)+x_{1}^{*}(k)\right)^{2}}\right] N_{1}(k)\right. \\
& +\left[\frac{x_{2}^{*}(k)\left(1-a_{21}(k) x_{1}^{*}(k)-a_{23}(k) x_{3}^{*}(k)\right)}{m_{12}(k) x_{2}^{*}(k)+x_{1}^{*}(k)}-\frac{a_{21}(k) a_{23}(k) x_{1}^{*}(k) x_{2}^{*}(k) x_{3}^{*}(k)}{\left(m_{12}(k) x_{2}^{*}(k)+x_{1}^{*}(k)\right)^{2}}\right] \frac{N_{2}(k)}{x_{2}^{*}(k)} \\
& \left.+\left[\frac{a_{21}(k) m_{23}(k) x_{3}^{*}(k)}{\left(m_{12}(k) x_{2}^{*}(k)+x_{1}^{*}(k)\right)^{2}}-\frac{m_{23}(k)}{m_{23}(k) x_{3}^{*}(k)+x_{3}^{*}(k)}\right] N_{3}(k)+\rho_{2}\right\} x_{2}^{*}(k+1), \\
& N_{3}(k+1)=x_{3}(k+1)-x_{3}^{*}(k+1) \\
& =x_{3}(k) \exp \left\{-a_{3}(k)+\frac{a_{32}(k) x_{2}(k)}{m_{23}(k) x_{3}(k)+x_{2}(k)}\right\} \\
& -x_{3}^{*}(k) \exp \left\{-a_{3}(k)+\frac{a_{32}(k) x_{2}^{*}(k)}{m_{23}(k) x_{3}^{*}(k)+x_{2}^{*}(k)}\right\} \\
& =\left\{x_{3}(k) \exp \left[a_{32}(k)\left(\frac{x_{2}(k)}{m_{23}(k) x_{3}(k)+x_{2}(k)}-\frac{x_{2}^{*}(k)}{m_{23}(k) x_{3}^{*}(k)+x_{2}^{*}(k)}\right)\right]-x_{3}^{*}(k)\right\} \frac{x_{3}^{*}(k+1)}{x_{3}^{*}(k)} \text {, } \\
& =\left\{\left[a_{32}(k)-\frac{a_{32}(k) x_{2}^{*}(k)}{m_{23}(k) x_{3}^{*}(k)+x_{2}^{*}(k)}\right] N_{2}(k)+\left[\frac{a_{32}(k) x_{2}^{*}(k) x_{3}^{*}(k)}{m_{23}(k) x_{3}^{*}(k)+x_{2}^{*}(k)}\right.\right. \\
& \left.\left.-\frac{a_{32}(k) x_{2}^{*}(k) x_{3}^{*}(k)(k) m_{23}(k)}{\left(m_{23}(k) x_{3}^{*}(k)+x_{2}^{*}(k)\right)^{2}}\right] \frac{N_{3}(k)}{x_{3}^{*}(k)}+\rho_{3}\right\} x_{3}^{*}(k+1) \text {, }
\end{aligned}
$$


where $\left|\rho_{i}\right| /\|N\|(i=1,2,3)$ converges, uniformly with respect to $k \in Z^{+}$, to zero as $\|N\| \longrightarrow 0$.

Define Lyapunov function as follows:

$$
V(N(k))=\delta_{1}\left|\frac{N_{1}(k)}{x_{1}^{*}(k)}\right|+\delta_{2}\left|\frac{N_{2}(k)}{x_{2}^{*}(k)}\right|+\delta_{3}\left|\frac{N_{3}(k)}{x_{3}^{*}(k)}\right|,
$$

where $\delta_{1}, \delta_{2}$, and $\delta_{3}$ are positive constants given in (67)-(69), respectively, and satisfy $A_{i}^{*}>0(i=1,2,3)$. Calculating the difference of $V$ along the solution of systems (60)-(62) and using (i), (ii), and (iii), we have

$$
\begin{aligned}
\Delta V= & \delta_{1}\left|\frac{N_{1}(k+1)}{x_{1}^{*}(k+1)}-\frac{N_{1}(k)}{x_{1}^{*}(k)}\right|+\delta_{2}\left|\frac{N_{2}(k+1)}{x_{2}^{*}(k+1)}-\frac{N_{2}(k)}{x_{2}^{*}(k)}\right|+\delta_{3}\left|\frac{N_{3}(k+1)}{x_{3}^{*}(k+1)}-\frac{N_{3}(k)}{x_{3}^{*}(k)}\right| \\
\leq & -\delta_{1}\left\{\left[a_{11}(k)+\frac{a_{12}(k) x_{2}^{*}(k)}{x_{1}^{*}(k)\left(m_{12}(k) x_{2}^{*}(k)+x_{1}^{*}(k)\right)}-\frac{a_{12}(k) x_{2}^{*}(k)}{\left(m_{12}(k) x_{2}^{*}(k)+x_{1}^{*}(k)\right)^{2}}\right]\left|N_{1}(k)\right|\right. \\
& +\delta_{1} \frac{a_{12}(k)}{m_{12}(k) x_{2}^{*}(k)+x_{1}^{*}(k)}\left|N_{2}(k)\right| \\
& -\delta_{2}\left[\frac{a_{21}(k) a_{23}(k) x_{1}^{*}(k) x_{3}^{*}(k)}{\left(m_{12}(k) x_{2}^{*}(k)+x_{1}^{*}(k)\right)^{2}}-\frac{\left(1-a_{21}(k) x_{1}^{*}(k)-a_{23}(k) x_{3}^{*}(k)\right)}{m_{12}(k) x_{2}^{*}(k)+x_{1}^{*}(k)}\right]\left|N_{2}(k)\right| \\
& +\delta_{2}\left|\left[\frac{a_{21}(k)}{m_{12}(k) x_{2}^{*}(k)+x_{1}^{*}(k)}-\frac{a_{21}(k) x_{1}^{*}(k)}{\left(m_{12}(k) x_{2}^{*}(k)+x_{1}^{*}(k)\right)^{2}}\right]\right|\left|N_{1}(k)\right| \\
& +\delta_{2}\left|\left[\frac{a_{21}(k) m_{23}(k) x_{3}^{*}(k)}{\left(m_{12}(k) x_{2}^{*}(k)+x_{1}^{*}(k)\right)^{2}}-\frac{m_{23}(k)}{m_{23}(k) x_{3}^{*}(k)+x_{3}^{*}(k)}\right]\right|\left|N_{3}(k)\right| \\
& -\delta_{3}\left[\frac{a_{32}(k) x_{2}^{*}(k)(k) m_{23}(k)}{\left(m_{23}(k) x_{3}^{*}(k)+x_{2}^{*}(k)\right)^{2}}-\frac{a_{32}(k) x_{2}^{*}(k)}{m_{23}(k) x_{3}^{*}(k)+x_{2}^{*}(k)}\right]\left|N_{3}(k)\right| \\
& +\delta_{3}\left|\left[a_{32}(k)-\frac{a_{32}(k) x_{2}^{*}(k)}{m_{23}(k) x_{3}^{*}(k)+x_{2}^{*}(k)}\right]\right|\left|N_{2}(k)\right|+\sum_{i=1}^{3} \delta_{i}\left|\rho_{i}\right| \\
& -A_{1}\left|N_{1}(k)\right|-A_{2}\left|N_{2}(k)\right|-A_{3}\left|N_{3}(k)\right|+\sum_{i=1}^{3} \delta_{i}\left|\rho_{i}\right|,
\end{aligned}
$$

where

$$
\begin{aligned}
A_{1}= & \delta_{1}\left[a_{11}(k)+\frac{a_{12}(k) x_{2}^{*}(k)}{x_{1}^{*}(k)\left(m_{12}(k) x_{2}^{*}(k)+x_{1}^{*}(k)\right)}-\frac{a_{12}(k) x_{2}^{*}(k)}{\left(m_{12}(k) x_{2}^{*}(k)+x_{1}^{*}(k)\right)^{2}}\right] \\
& +\delta_{2}\left|\left[\frac{a_{21}(k)}{m_{12}(k) x_{2}^{*}(k)+x_{1}^{*}(k)}-\frac{a_{21}(k) x_{1}^{*}(k)}{\left(m_{12}(k) x_{2}^{*}(k)+x_{1}^{*}(k)\right)^{2}}\right]\right|, \\
A_{2}= & \delta_{2}\left[\frac{a_{21}(k) a_{23}(k) x_{1}^{*}(k) x_{3}^{*}(k)}{\left(m_{12}(k) x_{2}^{*}(k)+x_{1}^{*}(k)\right)^{2}}-\frac{\left(1-a_{21}(k) x_{1}^{*}(k)-a_{23}(k) x_{3}^{*}(k)\right)}{m_{12}(k) x_{2}^{*}(k)+x_{1}^{*}(k)}\right] \\
& +\delta_{1} \frac{a_{12}(k)}{m_{12}(k) x_{2}^{*}(k)+x_{1}^{*}(k)}+\delta_{3}\left|\left[a_{32}(k)-\frac{a_{32}(k) x_{2}^{*}(k)}{m_{23}(k) x_{3}^{*}(k)+x_{2}^{*}(k)}\right]\right|, \\
A_{3}= & \delta_{3}\left[\frac{a_{32}(k) x_{2}^{*}(k)(k) m_{23}(k)}{\left(m_{23}(k) x_{3}^{*}(k)+x_{2}^{*}(k)\right)^{2}}-\frac{a_{32}(k) x_{2}^{*}(k)}{m_{23}(k) x_{3}^{*}(k)+x_{2}^{*}(k)}\right] \\
& +\delta_{2}\left|\left[\frac{a_{21}(k) m_{23}(k) x_{3}^{*}(k)}{\left(m_{12}(k) x_{2}^{*}(k)+x_{1}^{*}(k)\right)^{2}}-\frac{m_{23}(k)}{m_{23}(k) x_{3}^{*}(k)+x_{2}^{*}(k)}\right]\right| .
\end{aligned}
$$


From (64), we have

where

$\Delta V \leq-A_{1}^{*}\left|N_{1}(k)\right|-A_{2}^{*}\left|N_{2}(k)\right|-A_{3}^{*}\left|N_{3}(k)\right|+\sum_{i=1}^{3} \delta_{i}\left|\rho_{i}\right|$,

$$
\begin{aligned}
A_{1}^{*}= & \delta_{1}\left[a_{11}(k)+\frac{a_{12}(k) \exp \left(m_{2}\right)}{\exp \left(M_{1}\right)\left(m_{12}(k) \exp \left(M_{2}\right)+\exp \left(M_{1}\right)\right)}-\frac{a_{12}(k) \exp \left(M_{2}\right)}{\left(m_{12}(k) \exp \left(m_{2}\right)+\exp \left(m_{1}\right)\right)^{2}}\right] \\
& +\delta_{2}\left[\frac{a_{21}(k)}{m_{12}(k) \exp \left(m_{2}\right)+\exp \left(m_{1}\right)}-\frac{a_{21}(k) \exp \left(m_{1}\right)}{\left(m_{12}(k) \exp \left(M_{2}\right)+\exp \left(M_{1}\right)\right)^{2}}\right] \mid, \\
A_{2}^{*}= & \delta_{2}\left[\frac{a_{21}(k) a_{23}(k) \exp \left(m_{1}+m_{3}\right)}{\left(m_{12}(k) \exp \left(M_{2}\right)+\exp \left(M_{1}\right)\right)^{2}}-\frac{\left(1-a_{21}(k) \exp \left(m_{1}\right)-a_{23}(k) \exp \left(m_{3}\right)\right)}{m_{12}(k) \exp \left(M_{2}\right)+\exp \left(M_{1}\right)}\right] \\
& +\delta_{1} \frac{a_{12}(k)}{m_{12}(k) \exp \left(m_{2}\right)+\exp \left(m_{1}\right)}+\delta_{3}\left|\left[a_{32}(k)-\frac{a_{32}(k) \exp \left(m_{2}\right)}{m_{23}(k) \exp \left(M_{3}\right)+\exp \left(M_{2}\right)}\right]\right|, \\
A_{3}^{*}= & \delta_{3}\left[\frac{a_{32}(k) \exp \left(m_{2}\right) m_{23}(k)}{\left(m_{23}(k) \exp \left(M_{3}\right)+\exp \left(M_{2}\right)\right)^{2}}-\frac{a_{32}(k) \exp \left(M_{2}\right)}{m_{23}(k) \exp \left(m_{3}\right)+\exp \left(m_{2}\right)}\right] \\
& +\delta_{2}\left|\left[\frac{a_{21}(k) m_{23}(k) \exp \left(M_{3}\right)}{\left(m_{12}(k) \exp \left(m_{2}\right)+\exp \left(m_{1}\right)\right)^{2}}-\frac{m_{23}(k)}{m_{23}(k) \exp \left(m_{3}\right)+\exp \left(m_{2}\right)}\right]\right| .
\end{aligned}
$$

Since $\left|\rho_{i}\right| /\|N\|(i=1,2,3)$ converges, uniformly with respect to $k \in Z^{+}$, to zero as $\|N\| \longrightarrow 0$, it follows from conditions (i),(ii), and (iii) that there is a positive constant $\varphi$ such that, if $k$ is sufficiently large and $\|N\|<\varphi$, then

$$
\Delta V \leq-\frac{\eta}{3}\left\{\left|N_{1}(k)\right|+\left|N_{2}(k)\right|+\left|N_{3}(k)\right|\right\}=-\frac{\eta}{3}\|N\| .
$$

It follows from Freedman [22] that the trivial solutions of (60)-(62) is uniformly asymptotically stable. Thus, the solution $x^{*}=\left\{x^{*}(k)\right\}=\left\{\left(x_{1}^{*}(k), x_{2}^{*}(k), x_{3}^{*}(k)\right)^{T}\right\}$ of (3) is uniformly asymptotically stable. According to Wang and $\mathrm{Lu}$ [23], we can conclude that the positive periodic solution of (3) is globally asymptotically stable. The proof ends.

\section{Numerical Example}

Given a discrete model as follows:

$$
\left\{\begin{array}{l}
x_{1}(k+1)=x_{1}(k) \exp \left\{a_{1}(k)-a_{11}(k) x_{1}\left(k-\tau_{1}(k)\right)-\frac{a_{12}(k) x_{2}(k)}{m_{12}(k) x_{2}(k)+x_{1}(k)}\right\} \\
x_{2}(k+1)=x_{2}(k) \exp \left\{-a_{2}(k)+\frac{a_{21}(k) x_{1}\left(k-\tau_{2}(k)\right)}{m_{12}(k) x_{2}\left(k-\tau_{2}(k)\right)+x_{1}\left(k-\tau_{2}(k)\right)}-\frac{a_{23}(k) x_{3}(k)}{m_{23}(k) x_{3}(k)+x_{2}(k)}\right\} \\
x_{3}(k+1)=x_{3}(k) \exp \left\{-a_{3}(k)+\frac{a_{32}(k) x_{2}\left(k-\tau_{3}(k)\right)}{m_{23}(k) x_{3}\left(k-\tau_{3}(k)\right)+x_{2}\left(k-\tau_{3}(k)\right)}\right\}
\end{array}\right.
$$

where $\quad a_{1}(k)=0.8, a_{2}(k)=0.3, a_{3}(k)=0.4, a_{11}(k)=0.3+$ $0.2 \sin k \pi, a_{12}(k)=0.2+0.2 \sin k \pi, a_{21}(k)=0.8+0.4 \sin$ $k \pi, a_{23}(k)=0.6+0.4 \sin k \pi, a_{32}(k)=0.9+0.2 \sin k \pi, m_{12}$ $(k)=0.4+0.4 \sin k \pi, \quad m_{23}(k)=0.5+0.3 \sin k \pi$, and $\tau_{1}$ $(k)=\tau_{2}(k)=\tau_{3}(k)=1$. Then, $\bar{a}_{1}=0.8, \overline{\left(a_{12} / m_{12}\right)}=0.5$, $S_{1}=0.5823, \bar{a}_{11}=0.3, \bar{a}_{2}=0.3, \bar{a}_{21}=0.8, \bar{a}_{3}=0.4, c$ and $\bar{a}_{32}=0.9$. Thus, (H1)-(H3) hold. Therefore, system (71) has at least a positive two-periodic solution, which is shown in Figure 1.

\section{Data Availability}

No data were used to support this study.

\section{Conflicts of Interest}

The authors declare that they have no conflicts of interest. 


\section{Acknowledgments}

This work was supported by the National Natural Science Foundation of China (no. 61673008), Project of High-level Innovative Talents of Guizhou Province ([2016]5651), Major Research Project of the Innovation Group of the Education Department of Guizhou Province ([2017]039), Project of Key Laboratory of Guizhou Province with Financial and Physical Features ([2017]004), Hunan Provincial Key Laboratory of Mathematical Modeling and Analysis in Engineering (Changsha University of Science and Technology) (2018MMAEZD21), University Science and Technology Top Talents Project of Guizhou Province (KY[2018]047), Guizhou University of Finance and Economics (2018XZD01), and Key Project of Hunan Education Department (17A181).

\section{References}

[1] Z. Zhang and T. Tian, "Multiple positive periodic solutions for a generalized predator-prey system with exploited terms," Nonlinear Analysis: Real World Applications, vol. 9, no. 1, pp. 26-39, 2008.

[2] Z. Q. Zhang and H. L. Wang, "Existence and global attractivity of a positive periodic solution for a generalized prey-predator system with time delay," Mathematical and Computer Modelling, vol. 44, no. 1-2, pp. 188-203, 2006.

[3] Y. Li, K. Zhao, and Y. Ye, "Multiple positive periodic solutions of species delay competition systems with harvesting terms," Nonlinear Analysis: Real World Applications, vol. 12, no. 2, pp. 1013-1022, 2011.

[4] X. Ding, B. Su, and J. Hao, "Positive periodic solutions for impulsive Gause-type predator-prey systems," Applied Mathematics and Computation, vol. 218, no. 12, pp. 67856797, 2012.

[5] J. Shen and J. Li, "Existence and global attractivity of positive periodic solutions for impulsive predator-prey model with dispersion and time delays," Nonlinear Analysis: Real World Applications, vol. 10, no. 1, pp. 227-243, 2009.

[6] G. Zhu, X. Meng, and L. Chen, "The dynamics of a mutual interference age structured predator-prey model with time delay and impulsive perturbations on predators," Applied Mathematics and Computation, vol. 216, no. 1, pp. 308-316, 2010.

[7] K. Wang, "Existence and global asymptotic stability of positive periodic solution for a Predator-Prey system with mutual interference," Nonlinear Analysis: Real World Applications, vol. 10, no. 5, pp. 2774-2783, 2009.

[8] K. Wang, "Permanence and global asymptotical stability of a predator-prey model with mutual interference," Nonlinear Analysis: Real World Applications, vol. 12, no. 2, pp. 10621071, 2011.

[9] D. He, W. Huang, and Q. Xu, “The dynamic complexity of an impulsive Holling II predator-prey model with mutual interference," Applied Mathematical Modelling, vol. 34, no. 9, pp. 2654-2664, 2010.

[10] B. M. Al-eideh and A. J. Alawneh, "Asymptotic distribution of MLE's of the parameters for a diffusion model with catastrophes and some related inference problems," Journal of Interdisciplinary Mathematics, vol. 6, no. 1, pp. 19-26, 2003.

[11] T. Zhang and L. Xiong, "Periodic motion for impulsive fractional functional differential equations with piecewise
Caputo derivative," Applied Mathematics Letters, vol. 101, Article ID 106072, 2020.

[12] T. Zhang, L. Yang, and L. Xu, "Stage-structured control on a class of predator-prey system in almost periodic environment," International Journal of Control, vol. 93, no. 6, pp. 1442-1460, 2020.

[13] W. G. Kelly and A. C. Peterson, Difference Equations: An Introduction with Applications, Academic Press, Boston, MA, USA, 1991.

[14] R. Xu, H. Feng, P. Yang, and Z. Wang, "Persistence and stability in a ratio-dependent food-chain system with time delays," Applied Mathematics-A Journal of Chinese Universities, vol. 17, no. 1, pp. 39-47, 2002.

[15] R. P. Aagawal, Difference Equations and Inequalities: Theory, Methods and Applications, Monographs and Textbooks in Pure and Applied Mathematics, Vol. 228, Marcel Dekker, New York, NY, USA, 2000.

[16] J. D. Murry, Mathematical Biology, Biomathematics, Vol. 19, Springer, Berlin, Germany, 1989.

[17] B. X. Dai and J. Z. Zou, "Periodic solutions of a discrete-time nonautonomous predator-prey system with the BeddingtonDeAngelis functional response," Journal of Applied Mathematics and Computing, vol. 24, no. 1-2, pp. 127-139, 2007.

[18] R. Xu, L. Chen, and F. Hao, "Periodic solutions of a discrete time Lotka-Volterra type food-chain model with delays," Applied Mathematics and Computation, vol. 171, no. 1, pp. 91-103, 2005.

[19] J. Wiener, "Differential equations with piecewise constant delays, Trends in theory and practice of nonlinear differential equations," in Lecture Notes Pure Applied Mathematics, Marcel Dekker, New York, NY, USA, 1984.

[20] M. Fan and K. Wang, "Periodic solutions of a discrete time nonautonomous ratio-dependent predator-prey system," Mathematical and Computer Modelling, vol. 35, no. 9-10, pp. 951-961, 2002.

[21] R. E Gaines and J. L. Mawhin, Coincidence Degree and Nonlinear Differential Equations, Springer-Verlag, Berlin, Germany, 1997.

[22] H. I. Freedman, Deterministic Mathematical Models in Population Ecology, Monographs and Textbooks in Pure and Applied Mathematics, Marcel Dekker, New York, NY, USA, 1980.

[23] W. D. Wang and Z. Y. Lu, "Global stability of discrete models of Lotka-Volterra type," Nonlinear Anal: TMA.vol. 35, no. 9, pp. 1019-1030, 1999. 